\title{
Irregular arrangement of collecting venules (IRAC) provides a critical endoscopic insight in Helicobacter pylori-induced gastritis: A secondary publication
}

\author{
YOSHIKI KATAKE $^{1 *}$, KAZUHITO ICHIKAWA ${ }^{2 *}$, CHIKAU FUJIO ${ }^{3}$, SHIGEKI TOMITA ${ }^{2}$, \\ JOHJI IMURA $^{4}$ and TAKAHIRO FUJIMORI ${ }^{2}$
}

${ }^{1}$ Katake Clinic (Ichouka Naika), Hyogo; ${ }^{2}$ Department of Surgical and Molecular Pathology,

Dokkyo Medical University School of Medicine, Tochigi; ${ }^{3}$ Fujio Clinic, Hyogo; ${ }^{4}$ Department of Diagnostic Pathology,

Graduate School of Medicine and Pharmaceutical Sciences, University of Toyama, Toyama, Japan

Received August 13, 2012; Accepted August 22, 2012

DOI: $10.3892 / b r .2012 .8$

\begin{abstract}
The aim of this study was to evaluate the significance of an endoscopic atrophic border and irregular arrangement of collecting venules (IRAC) in the diagnosis of Helicobacter pylori (H.pylori)-induced gastritis. Upper gastrointestinal tract endoscopy was performed on 723 patients, who were screened them for $H$. pylori infection. Any patients who had undergone $H$. pylori eradication therapy were excluded from the study. The endoscopic atrophic border and IRAC in each patient were assessed. The H. pylori status was determined in the patients by combination of a serological test and/or histopathological examination. The H. pylori infection rates were $95.4 \%$ (455/477) in the group with an endoscopic atrophic border and $22.3 \%(55 / 246)$ in the group without an endoscopic atrophic border. In the diagnostic validity check, presence of an endoscopic atrophic border had a sensitivity of $89.2 \%$ and a specificity of $89.7 \%$. Furthermore, the $H$. pylori infection rates were 95.5\% (506/530) in the IRAC group and $2.1 \%(4 / 193)$ in the regular arrangement of collecting venules (RAC) group. In the diagnostic validity check, IRAC had a sensitivity of $99.2 \%$ and a specificity of $88.7 \%$. In conclusion, the presence of an endoscopic atrophic border and IRAC are highly indicative of an $\mathrm{H}$. pylori-infected gastric mucosa.
\end{abstract}

Correspondence to: $\mathrm{Dr}$ Kazuhito Ichikawa, Department of Surgical and Molecular Pathology, Dokkyo Medical University School of Medicine, 880 Kitakobayasi, Mibu, Shimotsuga, Tochigi 321-0293, Japan

E-mail: i-kazu@dokkyomed.ac.jp

*Contributed equally

Key words: Helicobacter pylori, endoscopic diagnosis, irregular arrangement of collecting venules, regular arrangement of collecting venules

\section{Introduction}

Since the identification of Helicobacter pylori (H. pylori) in 1983 (1), the diagnosis and treatment of upper gastrointestinal diseases, such as gastritis, peptic ulcer, gastric carcinoma and mucosa-associated lymphoid tissue (MALT) lymphoma, have undergone changes (2-7). The condition of $H$. pylori-infected gastric mucosa is characterized as acute or chronic inflammation, mucosal atrophy and intestinal metaplasia (8-10). The endoscopic features of $H$. pylori-induced gastritis include erythema and erosions, neither of which are specific. Atrophic gastritis and intestinal metaplasia are also observed secondary to $H$. pylori infection, but these findings are not easy to correctly diagnose by conventional endoscopy. In the present study, H. pylori infection was evaluated in atrophic gastritis and its endoscopic features to determine whether H. pylori-infected gastric mucosa can be diagnosed through an endoscopically superficial vascular network.

\section{Materials and methods}

Patients. This study was performed according to the principles of the Declaration of Helsinki. Upper gastrointestinal tract endoscopy was performed on 723 patients (510 males and 213 females), who had been screened for $H$. pylori infection during the past one year at Katake and Fujio Clinic, Hyogo, Japan. Any patients who had undergone $H$. pylori eradication therapy were excluded. The patients provided written informed consent for participation in the study. For the ethical procedure, linkable anonymizing method was used to ensure study blindness was maintained. Samples used in this study comprised materials for biopsy obtained for diagnosis or treatment and not for research. Medical disadvantage or risk of the patients did not increase by patient participation in this study and was obtained strictly for analysis of information as part of therapeutic intervention.

H. pylori infection status. The H. pylori status was determined in patients who were subjected to a combined serological test and/or histopathological examination. Serum samples were 
also tested for total $H$. pylori antibodies using the Pyloriset Dry (Orion Diagnostica, Espoo, Finland) latex agglutination test. Multiple gastric biopsy specimens were removed for histopathological examination $(11,12)$. To detect $H$. pylori, the samples were stained with hematoxylin and eosin, together with any accompanying special stains (Giemsa, Warthin-Starry) and immunohistochemical stains (Fig. 1). The biopsies were examined independently by two pathologists (K.I. and T.F.) who were unaware of the serological $H$. pylori status. If the serological test and/or histopathological examination results of $H$. pylori were positive, patients were diagnosed as being infected with $H$. pylori.

Observation by endoscopy. Endoscopic observation using high-resolution electronic endoscopy with an endoscopic video information system (Olympus Optical Co., Ltd., Tokyo, Japan and Fujifilm Corporation, Saitama, Japan) was carried out by two endoscopists (Y.K. and C.F.) who were unaware of the serological $H$. pylori status. The patients were closely observed after undergoing routine endoscopic examination. An endoscopic atrophic border was regarded as present or absent according to the Kimura-Takemoto classification (13). Following conventional endoscopy, the observed morphology of the capillary network structure was divided into two patterns: RAC, regular arrangement of collecting venules (Fig. 2) and IRAC, irregular arrangement of collecting venules (Fig. 3) (14). A RAC pattern was defined as numerous minute red points of similar size present at regular intervals throughout the viewing area. By contrast, an IR AC pattern was defined as an irregular or absent distribution of red points.

Statistical analysis. The sensitivity, specificity, positive and negative predictive values, likelihood ratios and accuracy were calculated with standard formulas (15).

\section{Results}

H. pylori infection and endoscopic atrophic border. The $H$. pylori infection rates were $95.4 \%(455 / 477)$ in the group that had an endoscopic atrophic border [mean age \pm standard deviation (SD), 57.3 \pm 12.4 years] and $22.3 \%(55 / 246)$ in the group without an endoscopic atrophic border (mean age $\pm \mathrm{SD}, 42.6 \pm 11.8$ ). In the diagnostic validity check, presence of an endoscopic atrophic border had a sensitivity of 89.2 and a specificity of $89.7 \%$. The positive predictive value was 95.4 , while the negative predictive value was $77.6 \%$. The positive likelihood ratio was 8.638 , while the negative likelihood ratio 0.120 . The accuracy was found to be $89.3 \%$ (Table I).

H. pylori infection and capillary network patterns. The H. pylori infection rates were $95.5 \%(506 / 530)$ in the IRAC group (mean age \pm SD, 56.2 \pm 13.2 ) and $2.1 \%$ (4/193) in the $\mathrm{RAC}$ group (mean age $\pm \mathrm{SD}, 48.9 \pm 12.9$ ). In the diagnostic validity check, IRAC had a sensitivity of $99.2 \%$ and a specificity of $88.7 \%$. The positive predictive value was $95.5 \%$, while the negative predictive value was $97.9 \%$. The positive likelihood ratio was 8.805 , while the negative likelihood ratio was 0.009 . The accuracy was found to be $96.1 \%$ (Table II).
Table I. Correlation between Helicobacter pylori infection and endoscopic atrophic border.

\begin{tabular}{lccc}
\hline & \multicolumn{2}{c}{ Helicobacter pylori infection } & \\
\cline { 2 - 3 } & Positive & Negative & Total \\
\hline Atrophic border & & & \\
Present & 455 & 22 & 477 \\
Absent & 55 & 191 & 246 \\
Total & 510 & 213 & 723 \\
\hline
\end{tabular}

Sensitivity $89.2 \%$, Specificity $89.7 \%$, positive predictive value $95.4 \%$, negative predictive value $77.6 \%$, positive likelihood ratio 8.638 , negative likelihood ratio 0.120 , accuracy $89.3 \%$.

Table II. Correlation between Helicobacter pylori infection and capillary network patterns.

\begin{tabular}{lrrr}
\hline & \multicolumn{2}{c}{ Helicobacter pylori infection } & \\
\cline { 2 - 3 } & Positive & Negative & Total \\
\hline IRAC & 506 & 24 & 530 \\
RAC & 4 & 189 & 193 \\
Total & 510 & 213 & 723
\end{tabular}

Sensitivity $99.2 \%$, specificity $88.7 \%$, positive predictive value $95.5 \%$, negative predictive value $97.9 \%$, positive likelihood ratio 8.805 , negative likelihood ratio 0.009 , accuracy $96.1 \%$. IRAC, irregular arrangement of collecting venules, RAC, regular arrangement of collecting venules.

\section{Discussion}

It would be useful to diagnose $H$. pylori status on the basis of endoscopic appearance alone in patients with $H$.pylori-related diseases such as gastritis, peptic ulcer, gastric carcinoma and MALT lymphoma (2-7). There has been some debate over whether $H$. pylori status can be diagnosed by endoscopy before biopsies and serological tests are performed (16-18). Previous studies have demonstrated that the extent of atrophic gastritis is a valuable endoscopic finding that helps in the diagnosis of $H$. pylori infection (8-10). Previously, Yagi et al $(14,19)$ reported that RAC in the gastric corpus seen by close observation essentially excluded $H$. pylori infection. There were also several reports that supported their seminal study (20-24). Moreover, it has been reported that magnifying narrow-band imaging (NBI) is useful for predicting $H$. pylori infection (25).

$H$. pylori infection can be diagnosed by two main methods. Invasive tests that require endoscopy and non-invasive or minimally invasive tests that do not require endoscopy. The invasive tests include rapid urease tests, culture, histopathological examination including immunohistochemistry and polymerase chain reaction (PCR)-based methods, while the non-invasive tests include serology, $H$. pylori stool antigen test and urea 

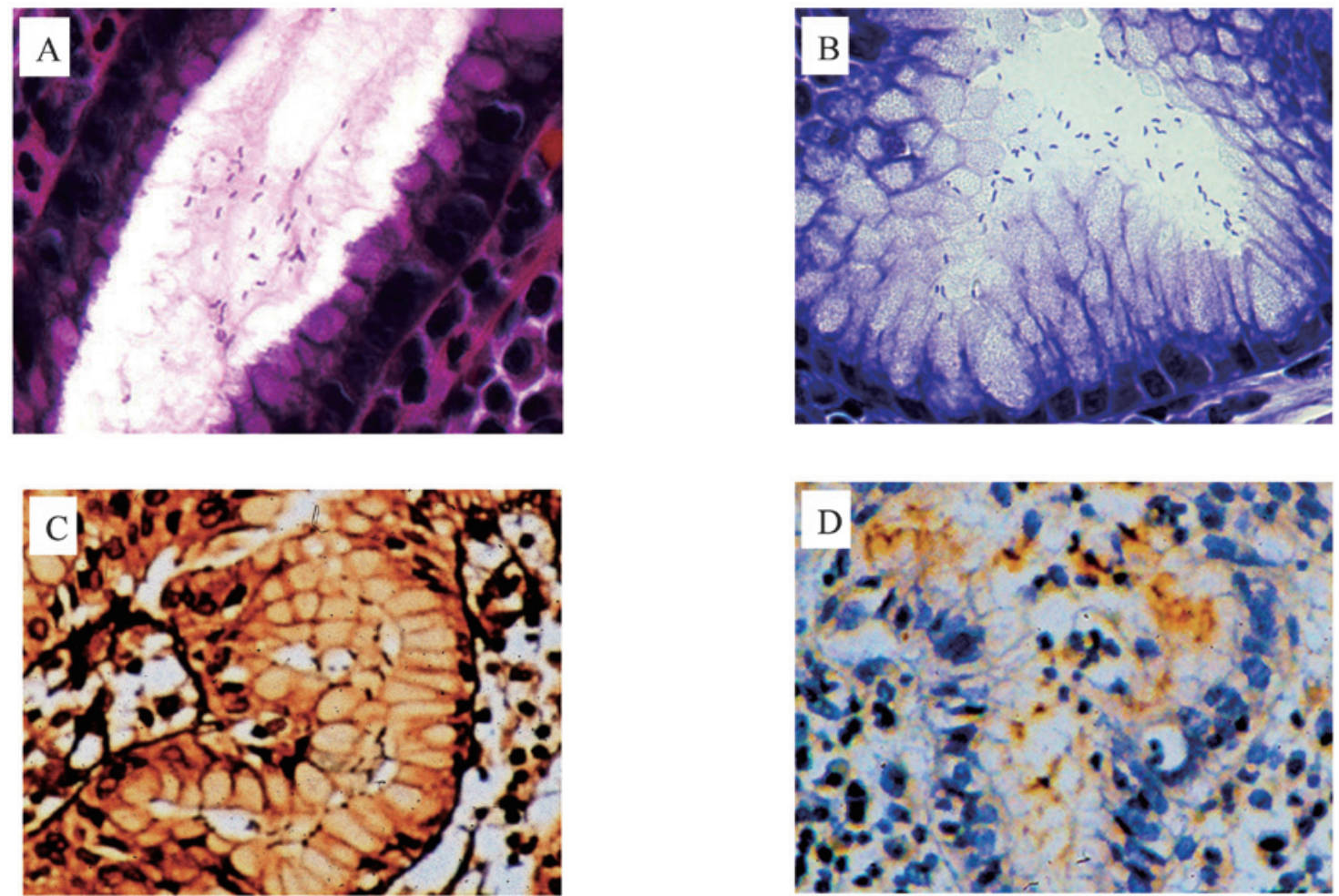

Figure 1. Histopathological detection of H. pylori in gastric biopsy specimen is shown. The organisms in the surface of gastric mucosa [(A), hematoxylin and eosin stain; (B), Giemsa stain; (C), Warthin-Starry stain]. (D), Immunohistochemical stain is focally positive.
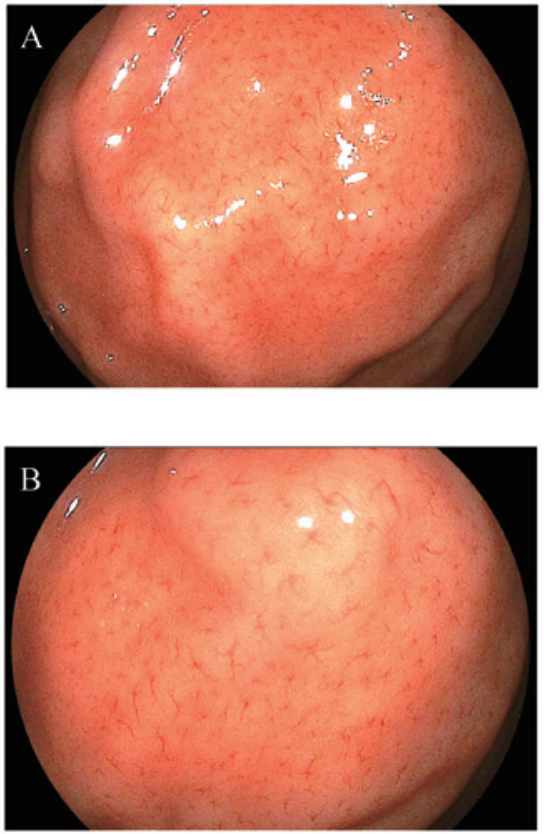

Figure 2. Typical endoscopic view of the regular arrangement of collecting venules (RAC) by high-resolution endoscopy [(B) was increased by magnification of (A) to obtain a more detailed view]. Courtesy of Dr C. Fujio, Fujio Clinic, Kobe, Hyogo.

breath test. With the exception of the PCR-based methods, these tests were recommended for the diagnosis of $H$. pylori infection prior and subsequent to eradication therapy in the guidelines for the management of $H$. pylori infection in Japan (26). However, the guidelines did not include a description of
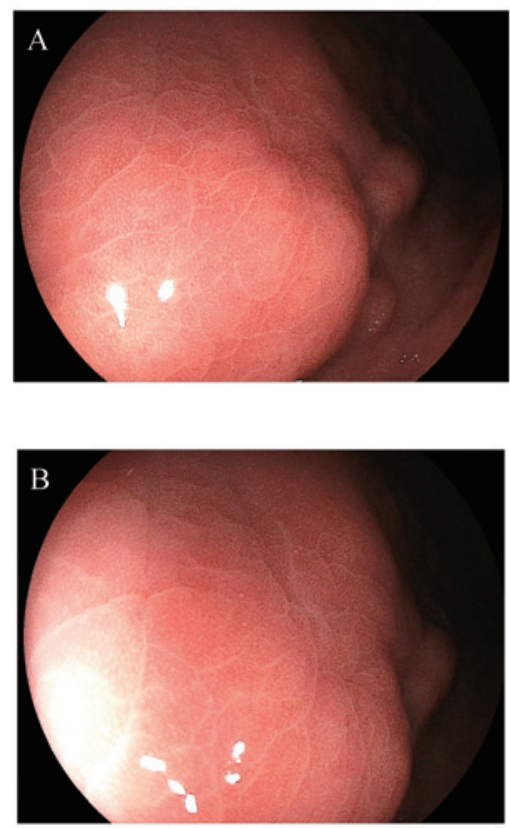

Figure 3. Typical endoscopic view of the irregular arrangement of collecting venules (IRAC) by high-resolution endoscopy; collecting venules cannot be seen [(B) was increased by magnification of $(A)$ to obtain a more detailed view]. Courtesy of Dr C. Fujio, Fujio Clinic, Kobe, Hyogo.

endoscopic findings that may be helpful in diagnosing H.pylori infection. The rapid urease test and histopathological examination with biopsy are accurate methods for identifying $H$. pylori. However, these methods are more invasive and expensive tests as compared to endoscopy without biopsy. 
In this study, we confirmed that there was good agreement between endoscopic findings and H.pylori status. Our results indicate that the presence of an endoscopic atrophic border and IRAC pattern were significant indicators of an $H$. pylori-infected gastric mucosa. Thus, these findings are the most reliable criteria for the diagnosis of $H$. pylori infection. The absence of an endoscopic atrophic border and/or the presence of a RAC pattern suggests that in such cases biopsy for histopathological examination and rapid urease test to detect $H$. pylori infection would be unnecessary. Therefore, we believe that $H$. pylori screening by endoscopic examination without biopsy is an excellent test of high diagnostic accuracy and cost-effectiveness.

In conclusion, the presence of an endoscopic atrophic border and IRAC are highly indicative of an H.pylori-infected gastric mucosa.

\section{Addendum}

This article is based on a study first reported in the Stomach and Intestine 2002; 37: 331-336 (Japanese paper with English abstract, non-inclusion of MEDLINE). Since the definition of regular arrangement of collecting venules (RAC) was not clearly determined in the first report, it was expressed as 'red spot pattern'. Therefore, we performed a re-design of this study, and attempted a secondary publication in English according to conditions for acceptable secondary publications as stated in Uniform Requirements for Manuscripts Submitted to Biomedical Journals (International Committee of Medical Journal Editors). Additionally, the results of the re-analysis, including additional cases from the Fujio clinic, were similar to those in the first report. Therefore, a secondary version of the initial report includes content that faithfully reflects the data of the primary version, and is not a 'meat-expander' article.

Duplicate publication has been an issue for debate worldwide. However, the importance of secondary publications has been suggested, mainly in Northern Europe. The World Medical Association has adopted the Declaration of Helsinki with regard to the ethics of research. As to the ethics of publication, however, the conditions for acceptable secondary publications are described in the Uniform Requirements for Manuscripts Submitted to Biomedical Journals: Writing and Editing for Biomedical Publication Updated April 2010 (http://www. icmje.org/) as follows: i) the authors have received approval from the editors of both journals (the editor concerned with the secondary publication is required to have a photocopy, reprint, or manuscript of the primary version), ii) the priority of the primary publication is respected by a publication interval of at least 1 week (unless specifically negotiated otherwise by both editors), iii) the paper for secondary publication is intended for a different group of readers; an abbreviated version is regarded as sufficient, iv) the secondary version faithfully reflects the data and interpretations of the primary version, v) the footnote on the title page of the secondary version informs readers, peers, and documenting agencies that the paper has been published in whole or in part and states the primary reference. A suitable footnote might read: 'This article is based on a study first reported in the (title of journal, with full reference).' Permission for such secondary publication should be free of charge: vi) the title of the secondary publication should indicate that it is a secondary publication (complete republication, abridged republication, complete translation or abridged translation) of a primary publication. Of note, the National Library of Medicine (NLM) does not consider translations to be 'republications' and does not cite or index translations when the original article was published in a journal that is indexed in MEDLINE, vii) editors of journals that simultaneously publish in multiple languages should understand that NLM indexes the primary language version. When the full text of an article appears in more than one language in a journal issue (such as Canadian journals with the article in both English and French), both languages are indicated in the MEDLINE citation. These conditions have been accepted widely in academic journals and they would also be adopted in this journal. This report clarified conditions for acceptable secondary publication in this journal. Significance of secondary publication should be considered from an international perspective according to the rule previously described, instead of the manner as in the proverb: 'A scalded cat fears cold water', in order for a study to be appraised internationally.

\section{Acknowledgements}

The authors would like to thank Dr Ichiro Hirata (Department of Gastroenterology, Fujita Health University Hospital, Aichi, Japan), Dr Chikao Shimamoto and Dr Kenichi Katsu (Second Department of Internal Medicine Osaka Medical College, Osaka, Japan) for their help in the first Japanese study. In addition, we thank Chiaki Matsuyama, Ayako Shimizu, Midori Katayama, Atsuko Kikuchi and Shizuka Kidachi (Department of Surgical and Molecular Pathology, Dokkyo University School of Medicine, Tochigi, Japan) for their excellent technical and secretarial assistance.

\section{References}

1. Marshall B and Warren JR: Unidentified curved bacilli in the stomach of patients with gastritis and peptic ulceration. Lancet 1: 1311-1315, 1984.

2. NIH consensus conference. Helicobacter pylori in peptic ulcer disease. NIH consensus development panel on Helicobacter pylori in peptic ulcer disease. JAMA 272: 65-69, 1994.

3. Uemura N, Okamoto S, Yamamoto S, et al: Helicobacter pylori infection and the development of gastric cancer. N Engl J Med 345: 784-789, 2001.

4. Parsonnet J, Friedman GD, Vandersteen DP, et al: Helicobacter pylori infection and the risk of gastric carcinoma. N Engl J Med 325: 1127-1131, 1991.

5. Huang JQ, Sridhar S, Chen Y and Hunt RH: Meta-analysis of the relationship between Helicobacter pylori seropositivity and gastric cancer. Gastroenterology 114: 1169-1179, 1998.

6. Blaser MJ and Parsonnet J: Parasitism by the 'slow' bacterium Helicobacter pylori leads to altered gastric homeostasis and neoplasia. J Clin Invest 94: 4-8, 1994.

7. Wotherspoon AC, Doglioni C, Diss TC, et al: Regression of primary low-grade B-cell gastric lymphoma of mucosa-associated lymphoid tissue type after eradication of Helicobacter pylori. Lancet 342: 575-577, 1993.

8. Mihara M, Haruma K, Kamada T, et al: The role of endoscopic findings for the diagnosis of Helicobacter pylori infection: evaluation in a country with high prevalence of atrophic gastritis. Helicobacter 4: 40-48, 1999.

9. Satoh K, Kimura K, Taniguchi Y, et al: Distribution of inflammation and atrophy in the stomach of Helicobacter pylori-positive and -negative patients with chronic gastritis. Am J Gastroenterol 91: 963-969, 1996. 
10. Kawaguchi H, Haruma K, Komoto K, et al: Helicobacter pylori infection is the major risk factor for atrophic gastritis. Am J Gastroenterol 91: 959-962, 1996.

11. Ishida M, Terano A, Tabuchi M, et al: The comparison with rapid urease test and histological examination in detection of Helicobacter-pylori. Gastroenterol Endosco 40: 773-778, 1998.

12. Smith SB, Snow AN, Perry RL and Qasem SA: Helicobacter pylori: to stain or not to stain? Am J Clin Pathol 137: 733-838, 2012.

13. Kimura $\mathrm{K}$ and Takemoto T: An endoscopic recognition of the atrophic border and its significance in chronic gastritis. Endoscopy 3: 87-97, 1969.

14. Yagi K, Nakamura A and Sekine A: Characteristic endoscopic and magnified endoscopic findings in the normal stomach without Helicobacter pylori infection. J Gastroenterol Hepatol 17: 39-45, 2002.

15. Tanaka H, Ichikawa K, Fujimori T, et al: Diagnostic validity of DNMT-1 and 3b immunoreactivity in non-neoplastic epithelium of UC patients with and without neoplasia. DJMS 39: 29-35, 2012.

16. Laine L, Cohen H, Sloane R, et al: Interobserver agreement and predictivevalue of endoscopic findings for $H$. pylori and gastritis in normal volunteers. Gastrointest. Endosc 42: 420-423, 1995.

17. Khakoo SI, Lobo AJ, Shepherd NA and Wilkinson SP: Histological assessment of the Sydney classification of endoscopic gastritis. Gut 35: 1172-1175, 1994.

18. Bah A, Saraga E, Armstrong D, et al: Endoscopic features of Helicobacter pylori-related gastritis. Endoscopy 27: 593-596, 1995.

19. Yagi K, Aruga Y, Nakamura A and Sekine A: Regular arrangement of collecting venules (RAC): a characteristic endoscopic feature of Helicobacter pylori-negative normal stomach and its relationship with esophago-gastric adenocarcinoma. J Gastroenterol 40: 443-452, 2005.
20. Nakayama Y, Horiuchi A, Kumagai T, et al: Discrimination of normal gastric mucosa from Helicobacter pylori gastritis using standard endoscopes and a single observation site: studies in children and young adults. Helicobacter 9: 95-99, 2004.

21. Anagnostopoulos GK, Yao K, Kaye P, et al: High-resolution magnification endoscopy can reliably identify normal gastric mucosa, Helicobacter pylori-associated gastritis, and gastric atrophy. Endoscopy 39: 202-207, 2007.

22. Machado RS, Viriato A, Kawakami E and Patricio FR: The regular arrangement of collecting venules pattern evaluated by standard endoscope and the absence of antrum nodularity are highly indicative of Helicobacter pylori uninfected gastric mucosa. Dig Liver Dis 40: 68-72, 2008

23. Gonen C, Simsek I, Sarioglu S and Akpinar H: Comparison of high resolution magnifying endoscopy and standard videoendoscopy for the diagnosis of Helicobacter pylori gastritis in routine clinical practice: a prospective study. Helicobacter 14: 12-21, 2009.

24. Alaboudy A, Elbahrawy A, Matsumoto S, et al: Regular arrangement of collecting venules: Does patient age affect its accuracy? World J Gastrointest Endosc 3: 118-123, 2011.

25. Okubo M, Tahara T, Shibata T, et al: Usefulness of magnifying narrow-band imaging endoscopy in the Helicobacter pylori-related chronic gastritis. Digestion 83: 161-166, 2011.

26. Asaka M, Kato M, Takahashi S, et al: Guidelines for the management of Helicobacter pylori infection in Japan: 2009 revised edition. Helicobacter 15: 1-20, 2010. 